\title{
Jules Dupuit (1804-1866) et l'utilité publique des transports, actualité d'un vieux débat
}

Conférence donnée aux membres de l'AHICF à l'occasion de leur assemblée générale, le 27 mars 2003

\section{François Vatin}

\section{(2) OpenEdition}

\section{Journals}

Édition électronique

URL : https://journals.openedition.org/rhcf/1916

DOI : 10.4000/rhcf.1916

Éditeur

Rails \& histoire

Édition imprimée

Date de publication : 1 juin 2003

Pagination : $39-56$

ISBN : 0996-9403

ISSN : 0996-9403

Référence électronique

François Vatin, "Jules Dupuit (1804-1866) et l'utilité publique des transports, actualité d'un vieux débat », Revue d'histoire des chemins de fer [En ligne], 27 | 2003, mis en ligne le 15 janvier 2015, consulté le 22 avril 2022. URL : http://journals.openedition.org/rhcf/1916 ; DOI : https://doi.org/ 10.4000/rhcf.1916 


\section{François VATIN}

\section{Jules Dupuit (1804-1866) et I'utilité publique des transports, actualité d'un vieux débat}

Conférence donnée aux membres de I'AHICF àl'occasion de leur

assemblée générale, le 27 mars 2003

Il y a comme une provocation à évoquer la figure de Jules Dupuit dans le cadre d'une association consacrée à l'histoire des chemins de fer. En effet, Jules Dupuit, qui compte sans doute parmi les plus connus des ingénieurs du corps des Ponts et Chaussées du XIX ${ }^{e}$ siècle, ne s'est jamais occupé, à la différence de beaucoup d'ingénieurs de son corps, du secteur ferroviaire, alors même que sa carrière se situe durant la période cruciale du développement des chemins de fer en France. Ses spécialités furent principalement l'entretien des routes, l'hydraulique et la construction d'ouvrages d'art.

Mais Dupuit est surtout connu des économistes pour un mémoire publié en 1844 qui l'a fait considérer comme un «précurseur» de l'économie mathématique «néoclassique» de la fin du XIX ${ }^{\mathrm{e}}$ siècle et comme une figure marquante de la tradition des «ingénieurséconomistes » propre à l'histoire de la pensée économique française ${ }^{1}$. C'est par ce biais que son héritage théorique intéresse directement l'histoire des chemins de fer. En effet, sa théorie économique a alimenté les débats sur la question de la « tarification », si importante dans l'histoire de l'économie publique. Mais cette théorie est aussi à la source d'une réflexion sur les choix d'investissements dans les infrastructures de transport: hier l'« utilité » de la construction des chemins de fer ; aujourd'hui, plutôt, le choix de leur maintien.

1- Jules Dupuit, «De l'utilité des travaux publics», Annales des Ponts et Chaussées, 1844, $2^{\mathrm{e}}$ semestre, p. 332-375. Voir les commentaires classiques de ce mémoire in François Etner, Histoire du calcul économique en France, Paris, Economica, 1987 et Robert H. Ekelund et Robert Hebert, Secrets Origins of Modern Microeconomics. Dupuit and the Engineers, Chicago and London, University of Chicago Press, 1999, qui fait la synthèse d'un riche ensemble d'articles de ces deux auteurs. Ma propre interprétation figure in Bernard Grall : « La machine et l'impôt : Jules Dupuit, l'économie politique et la mécanique industrielle» (1997), repris in Jean-Pascal Simonin et François Vatin (sous la dir. de), L'Fuvre multiple de Jules Dupuit, Angers, Presses de l'Université d'Angers, 2002. 
Cet exposé s'appuie sur un ensemble de travaux originaux menés ces dernières années sur Jules Dupuit et, plus généralement, sur la tradition économique des corps des Ponts ${ }^{2}$. Il entend d'abord fournir de cet auteur une image plus complexe que celle qui a en général été retenue. Il vise ensuite à répondre la question suivante : pourquoi est-ce un ingénieur spécialiste de la route et non du fer qui a mené parmi les ingénieurs des ponts du Xix ${ }^{e}$ siècle la réflexion la plus profonde sur la formation de la valeur et des prix ? J'essayerai de montrer que c'est précisément parce que Dupuit raisonnait dans une économie sans marché (l'usage de la route en France au XIX siècle était libre) qu'il fut conduit à penser théoriquement la valeur, là où ses collègues des chemins de fer la voyaient se former «spontanément» dans les comptes d'exploitation des compagnies. Je conclurai sur l'actualité de cette pensée alors que, de nouveau, on s'interroge aujourd'hui sur les limites de l'évaluation économique par le marché.

\section{Jules Dupuit : vie et œuvre}

Né en 1804, entré à Polytechnique en 1822, Jules Dupuit a fait toute sa carrière dans le corps des Ponts et Chaussées. Il fut successivement en poste dans la Sarthe (1827), la Marne (1840), le Maine-et-Loire (1844), puis enfin à Paris (1850) où il dirigea le service de la voirie, ce qui lui valut quelque démêlés avec le baron Haussmann. Nommé en 1855 inspecteur général, il siégea jusqu’à sa mort en 1866 au Conseil général des Ponts et Chaussées. Son parcours constitue, en somme, une carrière ordinaire d'ingénieur des Ponts du XIx ${ }^{e}$, un peu lente au début, particulièrement brillante à la fin, puisque tous, loin de là, ne finissaient pas au Conseil général ${ }^{3}$.

\footnotetext{
2- Outre l'ouvrage cité dans la note supra, je me réfère ici au travail considérable réalisé par Bernard Grall (décédé en 1997) et édité par mes soins avec l'aide de L'AHICF : Économie de forces et production d'utilité : la pensée gestionnaire des ingénieurs des Ponts et Chaussées (1831-1891), à paraitre aux Presses universitaires de Rennes avec le soutien de la Fondation Carnot, 2003.

3- Voir sur le corps des ponts au XIX siècle A. Brunot et R. Coquant, Le Corps des Ponts et Chaussées, Paris, éditions du CNRS, 1982 ; Antoine Picon, L'Invention de l'ingénieur moderne. L'École des Ponts et Chaussées (1747-1851), Paris, Presses de l'École nationale des Ponts et Chaussées, 1992.
} 
Jules Dupuit s'est donc occupé principalement de gestion routière, de construction et de restauration d'ouvrages d'art ${ }^{4}$, d'adduction d'eau et de gestion des égouts, d'aménagement urbain. A la différence de beaucoup d'ingénieurs des sa génération, il n'a jamais à proprement parler travaillé dans le secteur ferroviaire, nous l'avons dit. Comme beaucoup d'ingénieurs polytechniciens du XIX ${ }^{\mathrm{e}}$ siècle, c'est aussi un « savant ", c'est-à-dire qu'il ne se contentait pas de faire, mais qu'il cherchait aussi à construire les instruments théoriques de sa pratique. Sa science, parfois très développée, en mécanique comme en économie, est donc toujours engagée dans l'action.

Dès 1833, alors qu'il est en poste dans la Sarthe, Dupuit mène des expériences sur le frottement du roulement, question technique essentielle pour la gestion routière. Ces travaux ne sont pas qu'empiriques, puisqu'ils débouchent sur une théorie du frottement alternative à celle d'Arthur Morin - reprise de Charles Coulomb - qui fait alors autorité . En 1839, Dupuit est appelé à Paris pour poursuivre ses expériences. C'est l'origine de sa notoriété nationale dans le corps des Ponts. L'enjeu de cette discussion apparemment très technique est économique : il s'agit d'évaluer l'usure des chaussées induite par le passage des véhicules, usure qui exige, pour être compensée, un travail d'entretien routier à la charge de l'impôt $t^{6}$. Cette controverse scientifique sur le frottement s'inscrit donc dans un débat sur la " police du roulage » qui n'a pas cessé depuis la Révolution. Les questions qu'il pose sont: faut-il faire payer à l'usager l'usage de la voirie, comme en Angleterre ? Faut-il aussi, même si la route est gratuite, réglementer les formats des véhicules ? Et si oui, d'après quels principes ? Pour répondre à de telles questions, il faut mesurer précisément cette usure et dégager ses principales causes, c'est-à-dire les variables d'une "fonction d'usure ». Mais la question ne se limite pas à cette « économie naturelle » de la route. Car le trafic, cause de l'usure des chaussées, est aussi, en quelque sorte, le « produit », l'« utilité finale » du travail d'entretien routier. La principale cause de l'usure, à savoir l'intensité du trafic, est donc aussi l'objet même de la route.

4- Jules Dupuit s'est beaucoup occupé de construction et de réfection de ponts lors de son service dans le Maine-et-Loire ainsi que le relate Georges Reverdy, «Jules Dupuit, ingénieur en chef du Maine-et-Loire (1844-1850)» in J.-P. Simonin et F. Vatin, op. cit., p. 15-36. Il a rédigé un Traité de l'équilibre des voûtes et de la construction des ponts en maçonnerie, achevé après son décès par son disciple Mahyer.

5- Voir, sur le plan proprement scientifique, David Tabor, « A propos du frottement de roulement, une controverse oubliée ", Revue d'histoire des sciences, 1961, p. 13-18.

6- Je ne peux que brièvement relater ici des questions que l'on trouvera abondamment et finement analysées dans l'ouvrage à paraitre de Bernard Grall. 
On voit donc comment la pratique d'ingénieur met Dupuit, comme les autres ingénieurs de son corps, sur la voie de la théorie et du calcul économique. Le grand mémoire qu'il publie en 1842 sur «les frais d'entretien des routes » annonce directement celui bien plus connu de 1844 sur « la mesure de l'utilité des travaux publics » qui va retenir notre attention ${ }^{7}$. Dupuit signalait dans ce dernier mémoire le projet d'un ouvrage sur « l'économie publique appliquée aux travaux publics » qui ne verra jamais le jour, mais dont il publia des bribes dans divers articles. Ainsi, pour Dupuit, comme pour ses collègues des Ponts et des Mines, il n'existe pas de rupture franche entre le savoir mécanique, qui est à la base de leur pratique d'ingénieur, et l'économie proprement dite. Ce continuum entre la mécanique et l'économie est au principe même de la "mécanique industrielle », mécanique appliquée aux machines qui se développe dans les années 1820-1830 et qui constitue, tout au long du XIX ${ }^{e}$ siècle, la matrice théorique de l'ingénierie française $e^{8}$. Un de ses promoteurs, l'ingénieur des Mines Claude Burdin, l'affirmait sans fard en 1815 : «En rattachant ainsi la mécanique à l'économie politique, on montre le véritable point de vue selon lequel cette science doit être cultivée et envisagée?.»

Il faut souligner que cette référence à l'économie n'est pas un artifice rhétorique. Les ingénieurs français de la première moitié du XIX ${ }^{e}$ siècle lisent l'économie politique de leur temps. Ils connaissent Adam Smith, qu'ils citent souvent, parfois Sismondi, sur lequel Sadi Carnot a laissé des notes... Mais leur référence principale en la matière est JeanBaptiste Say, indiscutablement l'économiste le plus lu en France à cette époque et, notamment, par les ingénieurs. En ce qui concerne Dupuit, il faut citer également un autre nom: Pellegrino Rossi, immigré italosuisse qui fut appelé en 1834 par Guizot à prendre la succession de Jean-Baptiste Say à la chaire du collège de France ${ }^{10}$. Rossi, aujourd'hui

7- La même démonstration pourrait être faite dans le domaine de l'hydraulique et de la gestion des eaux sur laquelle Dupuit publie un ouvrage de synthèse en 1848 : Études théoriques et pratiques sur le mouvement des eaux courantes, Paris, Carilian-Goeury et Dalmont. Voir l'étude de cette partie de l'œuvre de Dupuit in Olivier Coutard et Kostas Chatzis, «Dupuit à propos du mode de distribution et du prix de vente de l'eau aux particuliers ", in J.-P. Simonin et F. Vatin, op. cit., p. 71-88.

8- J'ai développé ce point in F. Vatin, Le Travail, économie et physique (1780-1830), Paris, Puf, 1993.

9- Claude Burdin, «Considérations générales sur les machines en mouvement », Journal des mines, $\mathrm{n}^{\circ}$ 221, Paris, 1815.

10- Sur P. Rossi, voir F. Vatin, Economie politique et économie naturelle chę. A.A. Cournot, Paris, Puf, 1998, p. 103-127. Sur l'influence de Rossi sur Dupuit voir mes contributions ainsi que celles de Jean-Pascal Simonin dans notre ouvrage cité. 
très largement oublié, est un économiste original et puissant qui, dans le cours qu'il donna au Collège de France de 1834 à 1840, opéra une forme de synthèse de l'économie politique anglaise de David Ricardo et de celle, française, de Jean-Baptiste Say. Ces deux auteurs avaient débattu dans les années 1820 de la théorie de la valeur à travers les éditions successives de leurs œuvres respectives, mais aussi dans une correspondance personnelle nourrie. Or, la théorie de la valeur développée par Dupuit en 1844 procède directement de l'interprétation faite par Rossi de la controverse entre Say et Ricardo. Dupuit ne cessera de rendre hommage à Rossi tout au long de son œuvre économique, alors même que cet auteur n'était plus en cour dans le milieu des économistes libéraux français ${ }^{11}$.

L'économie politique constitue donc une langue largement partagée par les ingénieurs français de la génération de Dupuit. Elle est en permanence mobilisée dans leur pratique même d'ingénieur. Mais Dupuit s'est distingué en la matière de la plupart de ses collègues en devenant un «économiste » à proprement parler. Cette expression a alors un sens plus doctrinal qu'académique. Elle signifie l'adhésion à un groupe soudé dans une commune pensée : le libéralisme, et organisé dans une institution : la «Société d'économie politique », qui a son organe, le Journal des économistes, son éditeur attitré, Urbain Guillaumin, et qui tient des réunions mensuelles tous les quinze du mois auxquelles Dupuit fut assidu durant la dernière décennie de son existence ${ }^{12}$.

11- L'inspiration puisée par Dupuit chez Rossi est double. Elle est d'abord méthodologique : Rossi est le premier à avoir développé une opposition entre une économie politique « pure » ou « rationnelle » et une économie politique « appliquée », par analogie avec l'opposition entre la «mécanique rationnelle » et la "mécanique appliquée » (industrielle), mais aussi à celle entre le «droit rationnel» et le «droit positif». La possibilité de mathématiser le raisonnement économique repose sur une telle distinction que Léon Walras a reprise à la fin du xix ${ }^{e}$ siècle en s'inspirant notamment de Dupuit. Mais, par ailleurs, Rossi a indiscutablement inspiré directement Dupuit sur la théorie de la valeur. Curieusement, pourtant, ce dernier ne le cite pas en 1844, mais seulement en 1849 dans un autre mémoire qui répondait à la critique qui lui avait été faite en 1847 par son collègue Bordas dans les Annales des Ponts.

12- Dupuit ne fut assurément pas le seul ingénieur polytechnicien du XIX à faire œuvre d'économiste. Parmi ses aînés, on peut citer Achille Isnard, Charles Dupin ou ClaudeLucien Bergery ; parmi ses contemporains, Michel Chevalier, qui succéda à Pellegrino Rossi au Collège de France en 1840 ou Frédéric Le Play; parmi ses cadets, Émile Cheysson et Clément Colson, pour nous contenter d'une liste fort restreinte. 
A partir de 1849, Dupuit publie régulièrement dans le Journal des $e^{e}$ conomistes ${ }^{13}$. Il fournit d'abord des pièces de son ouvrage avorté sur «l'économie publique des travaux publics ». Il contribue de même en 1853 au Dictionnaire d'économie politique de Coquelin et Guillaumin par des articles sur son champ professionnel intitulés : « corps des ponts », « eau », " péage ", « routes et chemins », " voie de communication». On lui refuse en revanche l'article « utilité » qu'il avait écrit en reprenant ses thèses de 1844. Mais, dans les années 1860, Dupuit se tourne en économie vers des questions sans rapport avec son travail d'ingénieur. Il s'intéresse notamment aux «crises alimentaires » (1859), à la «liberté commerciale » (1861), à la propriété littéraire (1861), à la démographie (1865).

Dans ces divers travaux économiques, Dupuit fait figure de libéral passablement paradoxal. Dans un certain sens, il est « ultra-libéral », comme il l'était déjà en matière de gestion routière quand il prônait dans les années 1840 la liberté totale du roulage, c'est-à-dire non seulement l'absence de taxes, mais aussi celles de normes imposées aux véhicules ${ }^{14}$. De même, il se montre dans les années 1860 un libreéchangiste intransigeant et, en matière de crise alimentaire, critique toute forme d'intervention publique, soutenant que tout remède serait pire que le mal. En ce sens, il soutient souvent des thèses trop radicales pour ses collègues, plus pragmatiques, de la Société d'économie politique. Par exemple, il affirme que des faillites surviendront nécessairement avec l'introduction de la liberté commerciale et qu'elles sont utiles, puisque la mise en place d'une concurrence internationale est l'objet même d'une telle réforme. De même, il s'oppose, au nom d'une conception rigoureuse du principe de «liberté du travail », à la dépénalisation des coalitions ouvrières (loi Ollivier de 1864) que soutient en revanche la majorité des économistes libéraux du temps au nom du «droit des gens ». Enfin, il n’hésite pas à provoquer ses collègues de la Société d'économie politique en se prononçant contre le principe de chaires publique d'économie politique, jugeant que, pas plus là qu'ailleurs, la protection de l'État n'était légitime. En revanche, il reste un fervent défenseur du corps des Ponts et de ses prérogatives!

13- On trouvera une bibliographie développée de ses articles et de ses interventions en séance in J.-P. Simonin et F. Vatin, op. cit.

14- Son argumentaire reposait en la matière sur le modèle de la «main invisible» d'Adam Smith. Il était pour lui inutile d'imposer des normes aux transporteurs, car leur intérêt propre les conduisait spontanément à adopter les formats de véhicules et les chargements les plus avantageux pour la collectivité : ceux qui «fatiguaient» le moins la chaussée pour un tonnage transporté donné. 
Pourtant, selon moi, Dupuit n’est pas un « authentique libéral », au sens où il est d'abord et principalement un " utilitariste $»^{15}$. Le principe transcendant de liberté du sujet qui fonde pour la plupart des auteurs de son temps une morale libérale d'inspiration kantienne n'a aucune place dans sa philosophie économique. Il n'y a, pour lui, qu'une seule valeur sociale : l'utilité. L'augmentation de l'utilité collective justifie donc pleinement les restrictions à la liberté individuelle. La définition même de la société est pour lui cette restriction de la liberté individuelle au profit de l'utilité collective. Cette morale utilitariste se traduit de façon particulièrement suggestive dans les positions qu'il adopte sur le droit de propriété dans les années 1860. Alors que les libéraux cherchent à cette époque à donner, contre les socialistes, un fondement transcendant au droit de propriété, Jules Dupuit soutient, avec des accents qui évoquent parfois Marx, que cette démarche est vouée à l'échec. La propriété a pour lui toujours pour origine, proche ou lointaine, l'expropriation ; elle ne peut se justifier socialement que par son utilité. C'est pourquoi il lui semble qu'il faut protéger fortement la propriété foncière et son transfert par héritage, sans quoi le propriétaire n'investirait pas dans sa terre. En revanche, les droits de propriété intellectuelle et, notamment, de propriété littéraire doivent être modérés, afin que chacun puisse bénéficier de l'information qui est par nature un bien public $^{16}$. L'absence chez lui de toute conception transcendante du droit de propriété privée le conduit aussi à se montrer favorable à la nationalisation des entreprises en réseaux, telles les chemins de fer. Celles-ci constituent en effet des « monopoles naturels », c'est-à-dire que les rendements d'échelle y étant croissants, la concentration y est inévitable ; dans un tel contexte, mieux vaut que le monopole soit concédé à la puissance publique qu'à une entreprise privée.

La place de Dupuit dans l'histoire de la pensée économique est passablement paradoxale. Il n'est connu que pour son article de 1844 qui, à bien des égards, constitue encore un travail d'ingénieur. En revanche,

15- Voir sur ce point mon article, « La morale utilitaire de Jules Dupuit », in J.-P. Simonin et F. Vatin, op. cit., p. 91-116.

16- Selon la théorie économique un bien est " public » ou « collectif » si sa consommation par les uns n'entrave pas sa disponibilité pour les autres. De tels biens doivent être nécessairement financés par l'impôt. On distingue les biens collectifs "purs», pour lesquels il y a obligation d'usage (la police, la défense), et les biens collectifs « impurs », qui n'ont pas cette caractéristique et qui en conséquence peuvent être financés par des "péages ». (D’après Bernard Guerrien, Dictionnaire d'analyse économique, Paris, La Découverte, 2000, s.v. «Bien collectif ou bien public ») 
toute son œuvre postérieure, proprement économique, est restée fort peu commentée jusqu'à récemment. Mais le texte même de 1844 a fait l'objet d'une interprétation souvent abusive. Il a été lu en effet essentiellement comme un texte "précurseur» de la "révolution néoclassique $»^{17}$. Les économistes ont ainsi analysé ce texte sur la base de débats de trente ou quarante ans postérieurs au lieu de le situer dans son propre contexte. Replacer le texte de Dupuit dans son contexte suppose de pénétrer la pensée d'ingénieur pour elle-même en s'intéressant, notamment, à l'organisation de l'entretien des routes. Mais il faut aussi, pour comprendre la problématique de Dupuit, la situer par rapport aux discussions des économistes qu'avait lus Dupuit, tels Say et Rossi. C'est cette double opération de contextualisation que nous allons brièvement résumer maintenant.

\section{La mesure de I'utilité des travaux publics}

Toute la réflexion théorique de Dupuit en 1844 repose sur une discussion de la théorie de la valeur de Jean-Baptiste Say. Cette théorie de la valeur est fondée sur la notion d' "utilité », qui exprime l'apport subjectif d'un bien pour celui qui l'acquiert. Cette définition de la valeur par l'«aval » - la consommation - s'oppose à celle qui domine à la même époque l'économie politique anglaise issue de Ricardo, qui conçoit a contrario la source de la valeur en « amont» du processus économique, dans le coût de production du bien ${ }^{18}$. Contrairement à l'idée que l'on

17- On appelle « révolution néoclassique » ou «marginaliste » le renouvellement théorique en économie politique entamé dans le dernier tiers du XIX ${ }^{e}$ siècle par des auteurs comme Léon Walras en France, William Stanley Jevons en Angleterre et Karl Menger en Autriche. Ces diverses démarches simultanées se sont organisées dans un courant de pensée systématique qui a progressivement dominé la pensée économique moderne selon le schéma de la « révolution scientifique » conçu par Thomas Kuhn. Le changement de " paradigme » s'est incarné dans l'élaboration d'une théorie de la valeur fondée sur l'utilité marginale en lieu et place de la théorie classique du coût de production et sur la mathématisation systématique de la théorie économique. Les auteurs qui, tels Cournot ou Dupuit, avaient dans le courant du xix ${ }^{e}$ siècle approché de près ou de loin une telle doctrine ou développé des méthodes similaires sont alors devenus des " précurseurs ». Une telle représentation des choses, centrée sur le point focal que constituerait la théorie moderne, achevée, conduit souvent à simplifier outrageusement les choses en les expliquant par leur futur, plutôt que par leur présent. Elle ignore par exemple le fait que l'économie politique classique française, celle de Jean-Baptiste Say, reposait sur une théorie de la "valeur-utilité ».

18- Sur la controverse entre Say et Ricardo, voir F. Vatin, op. cit., 1998, p. 117-119 ainsi que "Pensée industrielle et théorie de la production chez Jean-Baptiste Say », in Jean-Pierre Potier et André Tiran (sous la dir. de), Jean-Baptiste Say. Nouveaux regards sur son ceuvre, Paris, Economica, 2002, p. 605-628. 
pourrait s'en faire a priori, la théorie de Say, qui s'inscrit dans une philosophie baconienne de la nature, est en phase avec la pensée des ingénieurs de son temps dont Say, professeur au Conservatoire des arts et métiers en compagnie de Charles Dupin, est en fait très proche ${ }^{19}$.

Jules Dupuit adopte, comme la plupart des auteurs français contemporains, cette théorie de la valeur-utilité. La discussion qu'il engage ne porte pas sur le concept d'utilité lui-même mais sur sa mesure. Say ne conçoit en effet l'utilité, du point de vue de la théorie économique, qu'en tant qu'elle est validée sur le marché par un prix. La valeur économique ne correspond pas au besoin qu'on a d'un bien dans l'absolu, mais au besoin solvable, à celui que l'on peut satisfaire pour le prix d'une somme d'argent. Pour Say, le prix de marché est donc la «mesure de l'utilité ». Que faire alors quand il n'y a pas de marché et donc pas de prix? C'est, suivant la terminologie moderne des économistes, la question des «biens publics ». Say est d'accord pour dire, par exemple, qu'une "promenade » apporte de l'utilité aux citadins, mais il considère «qu'un semblable avantage échappe à toute évaluation $»^{20}$. Une semblable réponse n'est toutefois pas possible quand il s'agit manifestement de biens de production, tels que les infrastructures de transport : routes, ponts ou canaux. Ceux-ci ont un coût (élevé), mais aussi une utilité dans un sens indiscutablement économique. Say admet d'ailleurs que « le service qu'en tire la société excède, dans la plupart des cas, la dépense annuelle qu'ils lui causent $»^{21}$.

Dans cette dernière formule, on voit poindre l'interrogation propre à Dupuit de la « mesure de l'utilité des travaux publics ». La question qu'il pose est celle, toujours contemporaine, de la légitimité des investissements publics. L'État ne peut légitiment financer la construction d'une route, d'un canal ou d'un pont que si, comme le formule Say, le «service productif » qu'en tire la société, autrement dit l'utilité publique de ce bien, excède son coût. Mais comment s'en assurer? Il faut pour cela trouver une mesure de l'utilité de ces biens collectifs, alors même que, comme on l'a vu, s'ils ont, en amont, un coût marchand, ils n'ont pas, en aval, de prix, puisque leur service n'est pas évalué par le consommateur à travers ses décisions d'achat. Say propose une solution à cette question, qui fut reprise par un ingénieur des Ponts aîné de Dupuit, Claude-Louis Navier (1785-1836) : l'utilité de tels biens publics de transport pourrait être mesurée par le différentiel des coûts supportés par

19- Voir sur ce point F. Vatin, op. cit., 1998.

20- J.-B. Say, Traité d'économie politique, 5e édition (1826), Paris, Calmann-Lévy, 1972, p. 506.

21- Ibidem, p. 508. 
les transporteurs. On peut calculer en effet le coût unitaire du transport entre deux points avant et après la mise en place de l'infrastructure considérée. L'utilité publique de cette infrastructure, c'est-à-dire le bénéfice qu'en tire la société, correspondrait donc à la différence de ces deux coûts multipliée par la quantité transportée.

Toute l'analyse de Dupuit part d'une critique de ce modèle d'évaluation. Il montre d'abord que Say n'est pas ici en accord avec ses principes, puisqu'il ne mesure pas directement l'utilité par la formation d'un prix marchand. Tout bien de production pourrait s'évaluer de la même manière. Or, en toute généralité, Say considère que l'utilité d'une machine quelconque est son prix de marché, comme il en est pour un bien de consommation. Il y a donc là un problème de cohérence théorique. Mais la critique de Dupuit est plus précise et, par là, plus incisive. Il note en effet que la mise en place d'une nouvelle infrastructure de transport va, en général, conduire à une augmentation du trafic. Si on adopte le modèle de Say et de Navier, il faudra appliquer à ces nouvelles unités transportées la valeur-utilité définie par la différence entre le prix ancien et le prix nouveau de transport. Or, précisément, ces biens nouvellement transportés ne l'étaient pas à l'ancien coût. Pourquoi ? Parce qu'ils n'en pouvaient payer le prix. C'est la baisse du prix de transport qui a rendu possible le nouveau trafic. Le modèle de SayNavier exagère donc l'utilité de la nouvelle infrastructure.

Pour comprendre la critique fondamentale adressée par Dupuit à Say, on peut faire un détour par la question de l'impôt indirect, soit celle de la « taxe » qui est au cœur de son argumentaire, car elle débouche sur son principe de tarification. Pour Say, une taxe ne crée pas de valeur, elle n'est qu'un " déplacement de richesse $»^{22}$. Sans doute, reconnait Dupuit ; nonobstant, souligne-t-il, elle met en évidence une « utilité cachée ", puisque le consommateur continue à acheter le produit taxé. Autrement dit, il donne au produit une utilité au moins égale à ce nouveau prix (le prix initial augmenté de la taxe). En conséquence, contrairement à ce que croit Say, le marché ne mesure pas l'utilité subjective des biens. Si un consommateur achète un bien c'est que la valeur de ce bien est, pour lui, au moins égale au prix qu'on lui en demande. Elle peut être plus élevée, puisqu'il est possible qu' il continue à l'acheter si on lui en demande un prix plus élevé, via une taxe par exemple : «Si vous prenez ce chiffre pour mesure, et non pas pour la limite inférieure

22- Jean-Baptiste Say distingue trois types de « déplacements de richesse » : l'impôt, le vol et la charité. 
d'une quantité dont vous ne connaissez pas la grandeur exacte, vous agissez comme un homme qui, voulant mesurer dans l'obscurité la hauteur d'un mur, et voyant qu'il ne peut en atteindre le sommet en élevant le bras, dirait : ce mur a deux mètres, car s'il n'avait pas deux mètres ma main eût passé par-dessus. Si vous dites : ce mur a au moins deux mètres, nous sommes d'accord ${ }^{23}$. "

Dupuit propose finalement une résolution générale du problème par ce que l'on pourrait appeler, selon la formule de l'épistémologue Ernst Mach, une «expérience de pensée ». La mesure de l'utilité d'un bien est, pour une personne donnée à un moment donné, le « prix maximum » que cette personne accepterait de payer ce bien. La théorie de Say reposait sur une mesure unique de l'utilité telle que l'établissait le prix de marché. La théorie de l'utilité de Dupuit est, elle, "différentielle ». L'utilité attribuée par chaque agent à un bien varie, suivant son revenu et, comme nous allons le voir, suivant la quantité de ce bien dont il dispose. Si, dans le cas général, le prix de marché est unique, la valeur, elle, est variable. Pour un consommateur donné, doté d'une quantité donnée de ce bien, cette valeur peut être inférieure au prix de marché (auquel cas il renonce à l'achat), ou, au contraire, supérieure à ce prix. En ce dernier cas, il dispose de ce qu'Alfred Marshall appellera une « rente », et Maurice Allais, grand amateur et commentateur moderne de Jules Dupuit, un « surplus ».

Cette théorie de la valeur, qui anticipe indiscutablement la pensée néoclassique, débouche sur la mise en évidence d'une « courbe de demande » que Dupuit trace dans les annexes de son mémoire. Cette courbe avait déjà mise en évidence en 1838 par Augustin Cournot ${ }^{24}$. Mais celui-ci ne s'appuyait pas sur une théorie de la valeur. Il considérait, à la différence de Dupuit, que l'utilité était par nature incommensurable. Il se contentait d'admettre l'hypothèse de bon sens selon laquelle la quantité achetée (le « débit » selon sa formule) augmentait quand le prix baissait et diminuait quand le prix augmentait. Le génie de Cournot est d'avoir formulé cette hypothèse banale, communément admise par les économistes du XVII ${ }^{\mathrm{e}}$ et du XIX ${ }^{\mathrm{e}}$ siècle, en une formule mathématique : $\mathrm{D}=\mathrm{f}(\mathrm{p})$, et d'en avoir tiré des conséquences logiques sans chercher à connaître les valeurs algébriques de cette fonction, ni même sa forme précise.

23- J. Dupuit, art. cit., 1844, p. 334.

24- A. Cournot, Recherches sur les principes mathématiques de la théorie des richesses (1838), Paris, Vrin, 1980 et mon commentaire sur cet ouvrage in F. Vatin, op. cit., 1998. 
Dupuit va moins loin que Cournot du point de vue de l'application du raisonnement mathématique aux grandeurs économiques. En revanche, il va plus loin que lui dans l'analyse théorique des fondements d'une telle fonction en la rattachant à sa conception de la variation de l'utilité, autrement dit à ce que les néoclassiques appelleront l' "utilité marginale », la « dérivée », au sens mathématique du terme, de l'utilité. Il montre en effet que, quand le prix baisse, de nouveaux consommateurs, mais aussi de nouveaux besoins solvables apparaissent. Ainsi, on utilisera l'eau, d'abord pour boire, puis pour se laver, pour nettoyer son intérieur, etc. L'analyse de l'utilité permet de mettre en évidence le « consommateur marginal », mais aussi la « consommation marginale ».

Quand on dispose d'une relation liant prix et quantités échangées, soit, selon la terminologie moderne, d'une « fonction de demande ", on peut calculer, comme le faisait déjà Cournot, un "prix de monopole », c'est-à-dire un prix qui maximise la recette du producteur. Si l'on néglige les coûts, il s'agit de trouver les coordonnées $\mathrm{D}$ et $\mathrm{p}$ du graphique liant ces deux grandeurs de telle sorte que le produit D.p soit maximal, autrement dit, géométriquement, le plus grand rectangle situé sous la courbe de demande ${ }^{25}$. Mais, en conformité avec sa théorie, Dupuit va au-delà de la figure du prix de monopole en proposant ce qu'on appellera une «tarification différentielle». Le principe en est de faire payer à chacun le prix maximum qu'il est prêt à accepter. Il faut pour cela "différencier le marché », comme on le fait en distinguant des « classes » de chemins de fer. La différence de prix ne résulte pas dans ce cas de la différence des coûts de production ; au contraire, on organise une variété de prestations, pour « différentier » la clientèle selon son revenu, c'est-à-dire selon sa capacité à payer le service.

Dupuit va très loin en ce domaine en défendant la «moralité » de cette logique économique contre l'éthique dominante des ingénieurs de son temps, pour qui le prix ne devait pas être rendu indépendant du coût de production. Il n'hésite pas ainsi à justifier ce qu'il appelle luimême la « ruse des commerçants » : «Cette valeur d'utilité si variable, si mobile, est au reste bien connue dans le commerce; et il y a longtemps qu'elle y est exploitée. [...] Il [le vendeur] a recours à une infinité de ruses

25- Cournot procède à une analyse fine en supposant d'abord le coût nul, puis un seul coût fixe, enfin un coût proportionnel à la quantité produite (c'est-à-dire un coût unitaire constant), enfin un coût variable mais fonction lui aussi de la quantité échangée. Dupuit, quant à lui, raisonne d'emblée, et sans justifier son propos, dans un modèle sans coût. Le bien (la route, le pont) est là et on néglige ses frais d'exploitation. Ce cadre de référence a priori manifeste bien sa conception d'une source de la valeur totalement indépendante des coûts de production. 
pour se faire payer à chacun d'eux [les acheteurs] la plus grande part possible de ce bénéfice ${ }^{26}$ qu'il considère comme fait à ses dépens. La même marchandise, déguisée dans divers magasins sous des formes variées, se vend souvent à des prix très différents aux gens riches, aux gens aisés et aux gens pauvres. Il y a le fin, le très-fin, le superfin, l'extrafin, qui, quoique sortis du même tonneau et ne présentant aucune différence réelle que celle du superlatif de l'étiquette, se vendent à des prix très différents. Pourquoi ? C'est que la même chose a une valeur d'utilité très différente suivant les consommateurs. Si l'on avait qu'un prix moyen, il y aurait perte pour tous ceux qui se priveraient de ce produit parce qu'ils attachent une utilité inférieure à ce prix, et perte pour le vendeur qui ne se ferait payer de beaucoup d'acheteurs qu'une trop petite partie de l'utilité du service rendu. A Dieu ne plaise que nous ne voulions justifier toute les fraudes du commerce ; mais il est bon de les étudier parce qu'elles sont fondées sur une connaissance exacte du cœur humain, et que dans beaucoup de cas on y trouve souvent plus d'équité qu'on ne s'y attendait d'abord, et même de bons exemples à suivre ${ }^{27}$. »

Il faut comprendre l'éthique économique de Dupuit, cyniquement exprimée, en rappelant sa morale utilitaire. Comme il le souligne, l'impôt qui rend un produit impossible à acheter pour certains pèse plus sur ceux qui ne le paient pas (ceux qui renoncent à l'achat) que sur ceux qui le payent. Faire payer à chacun le maximum qu'il accepte de payer permet ainsi d'augmenter la demande solvable, puisque chacun pourra acheter à proportion de ses moyens. Dupuit retrouve ici le fondement même de la théorie de la valeur utilité, formulé dès 1738 par Jacques Bernoulli dans sa résolution du paradoxe posé aux mathématiciens et philosophes de l'époque par un jeu d'argent, dit «jeu de Saint-Pétersbourg ${ }^{28}$ : une même somme d'argent n'a pas la même valeur pour chacun ; sa valeur relative dépend de la quantité qu'on en possède. Autrement dit, en langage moderne, l'utilité marginale de la monnaie est décroissante. Un tel raisonnement est celui qui fonde, par exemple, le principe de l'impôt progressif.

26- Dupuit évoque sous le nom de «bénéfice» ce que Maurice Allais appellera le « surplus du consommateur».

27- J. Dupuit, art. cit., 1844, p. 341-342.

28- Le paradoxe soumis aux mathématiciens était le suivant. La règle de ce jeu était telle que l' «espérance mathématique» de gain (probabilité de gain multipliée par ce gain) était infinie, alors même que, pour un joueur donné, il n'était manifestement pas raisonnable de poursuivre le jeu au-delà d'une certaine somme. Jacques Bernoulli déplace alors le problème en inventant le concept d' " espérance morale ", produit de la probabilité non par le gain absolu mais par le gain relatif (gain rapport à la fortune du joueur). 
Dupuit pense avoir trouvé ainsi un principe de tarification idéal : il maximise le profit du producteur à un degré supérieur encore au tarif de monopole mais, dans le même temps, il pousse à son maximum la demande solvable et, donc, l'utilité publique, puisque chacun possèdera le bien qu'il paiera à proportion de ses capacités. Un tel raisonnement n'est toutefois pas sans faiblesse. Il reste limité à un marché particulier ; c'est, autrement dit, un modèle d' « équilibre partiel ». Un producteur ne saurait en effet «soutirer» la rente du consommateur sur le marché d'un bien particulier sans provoquer des conséquences défavorables sur les autres marchés. Cette critique, qui se base sur le principe de l'interdépendance des marchés, fut formulée dès 1847 par un collègue de Dupuit, Bordas ${ }^{29}$. Elle sera reprise ultérieurement par Léon Walras, qui a laissé précisément son nom dans l'histoire de la pensée économique comme celui de l'inventeur de la « théorie de l'équilibre général » qui vise à concevoir les conditions de l'équilibre simultané sur tous les marchés.

\section{Le modèle de Dupuit et les chemins de fer}

Avant d'aborder ce dernier point de mon exposé, il me faut résumer les résultats précédents en revenant sur l'origine et sur la signification première du modèle élaboré par Dupuit en 1844. Son objectif premier n'était pas, comme on a pu le dire, la question de la tarification, ni même la théorie de la valeur. C'était, comme nous l'avons vu, la "mesure de l'utilité des travaux publics». Son interrogation se situe explicitement dans une réflexion sur les normes de gestion publique: comment évaluer l'intérêt public d'un investissement dans une infrastructure de transport, afin de garantir que son intérêt pour le public excédera le coût de l'investissement ? La réflexion profonde de Dupuit sur la théorie de la valeur découle de cette question pratique. Elle vient aussi, comme on l'a vu, de sa critique du modèle de Say et Navier qui surévaluait l'utilité des investissements dans les infrastructures de transport en donnant la même valeur à l'utilité du transport pour les quantités transportées avant la mise en place de la nouvelle infrastructure et pour les quantités nouvellement transportées, celles dont le transport résulte de la mise en place de cette infrastructure. C'est cette réflexion sur la valeur du transport qui a amené Dupuit à adopter un modèle différentiel et qui l'a mis sur la voie de la théorie de l'utilité marginale.

Ce problème de la mesure de l'utilité publique était bien sûr essentiel à un moment où la puissance publique investissait, directement

29- Voir B. Grall et F. Vatin, in J.-P. Simonin et F. Vatin, op. cit., p. 59-60. 
ou indirectement, des sommes considérables dans la construction des chemins de fer et autres grands travaux, avec les relents de scandale qui accompagnent inévitablement de telles opérations. Mais, nous l'avons dit, c'est à un ingénieur routier et non à un ingénieur ferroviaire que l'on doit la réflexion la plus riche sur cette question au XIx ${ }^{\mathrm{e}}$ siècle. Cet apparent paradoxe est riche de signification. Les chemins de fer sont concédés à des entreprises privées qui font payer la prestation de transport. C'est donc le marché qui va juger leur utilité. En résumé, un investissement est « rentable » s'il est profitable. Le calcul d'utilité marginale naît dans le secteur routier précisément du fait de l'absence d'un marché.

C'est donc en cherchant à résoudre une question pratique de gestion qui soulevait des difficultés particulières que Dupuit a renouvelé en profondeur l'analyse de la valeur. C'est en ce sens qu'il peut figurer à juste titre comme un « précurseur » des néoclassiques. Mais le problème que se pose Dupuit, comme le cadre général de sa solution, s’inscrivent dans l'espace paradigmatique de l'école classique française, marquée par ces deux sources fondamentales de Dupuit que sont Say et Rossi. Par ailleurs, comme j'y ai insisté, Dupuit ne peut dégager son principe théorique que parce qu'il adhère philosophiquement à une conception strictement utilitariste de la valeur. Selon cette philosophie, le coût ne saurait par lui-même justifier aucune valeur. La valeur réside, comme le posait bien Say, dans l'utilité. Le problème est qu'on sait mesurer le coût comme une somme monétaire, mais non l'utilité. La mesure qu'en proposait Say (le prix de marché) était manifestement insuffisante, voir incohérente. Dupuit complète donc l'économie de Say en lui fournissant un instrument théoriquement cohérent de mesure de l'utilité : le prix maximum accepté.

Le modèle de Dupuit débouche par ce biais sur une autre question qui intéresse directement l'économie ferroviaire alors en gestation : celle de la tarification. Cette question fait l'objet d'importants débats dans les Annales des Ponts des années 1840-1880. La morale économique spontanée des ingénieurs les amène pour la plupart à concevoir un principe de tarification fondé sur le coût : à coût égal, tarif égal. Mais comment mesurer ce qu'est un coût égal ? Dès lors qu'on sort de la pétition de principe pour réfléchir concrètement à partir de modèles de comptabilité analytique que les ingénieurs ferroviaires contribuent abondamment à concevoir durant cette période, à cette fin précisément, la question devient redoutablement complexe ${ }^{30}$. Parallèlement,

30- Voir sur cette question B. Grall, ouvrage à paraitre ainsi que François Caron, Histoire des chemins de fer en France, tome 1, 1740-1883, Paris, Fayard, 1997, p. 373 et sq. 
un autre principe de tarification se développe dans les chemins de fer: celui, loué par Dupuit, du commerçant qui cherche à obtenir de chaque client, de chaque transport, le maximum qu'il peut en tirer. J'ai déjà évoqué les «classes » pour le transport des voyageurs. Pour les marchandises, il s'agit notamment de la tarification «ad valorem » qui fait payer chaque marchandise à proportion de sa valeur, c'est-à-dire finalement, comme le souhaite Dupuit, à proportion de sa capacité à payer ${ }^{31}$.

Pourtant, curieusement, la théorie de Dupuit est, en apparence au moins, absente de ce débat. Sans doute a-t-il été lu par les ingénieurs des Ponts, car sa sévère controverse avec Bordas n’a pu passer inaperçue. Mais aucun article des Annales des Ponts ne le cite dans les années 1850-1880 alors que le débat sur la tarification bat son plein chez les ingénieurs ferroviaires. Ceux-ci se défendent en effet de mettre en place une tarification ad valorem, assimilée à un pouvoir de monopole. Quand les ingénieurs commencent à évoluer dans leur doctrine tarifaire au cours des années 1880, plutôt qu'à la théorie de Dupuit ils préfèrent se référer, pour légitimer une tarification plus « souple », à la « loi de l'offre et de la demande ». Comme il l'était pour les économistes libéraux de la Société d'économie politique, Dupuit représentait probablement un allié dangereux pour les ingénieurs ferroviaires partisans d'une tarification marchande, car il montrait trop clairement que la rationalité économique des compagnies n'était pas fondamentalement différente de celle du premier commerçant venu. Mais, surtout, dès lors que l'on disposait d'une légitimation par le marché, point n'était besoin d'aller chercher une légitimation théorique, complexe et sulfureuse. On démontre une nouvelle fois ici a contrario que c'est bien dans le cadre de l'économie routière et non dans celui de l'économie ferroviaire que la théorie de Dupuit pouvait éclore.

Ce n'est qu'à la fin des années 1880 que le mémoire de Dupuit commença à être débattu dans le milieu des ingénieurs des Ponts. Il faut dire qu'il avait alors reçu l'onction de la nouvelle école économique "néoclassique $»^{32}$. Mais c'est aussi qu'il entrait alors en résonance avec un problème nouveau rencontré par les compagnies au moment où le

31- Plus une marchandise a une valeur élevée et plus le surcoût du transport a, en valeur relative, un faible impact sur le prix final au consommateur.

32- Léon Walras, qui avait connu Jules Dupuit à la Société d'économie politique dans les années 1860 et appréciait manifestement peu la personne, chercha à minorer son importance dans la genèse de sa propre théorie, l'accusant, de façon totalement infondée, d'avoir en 1844 plagié Cournot. Cela lui valut une polémique avec William Stanley Jevons, le fondateur britannique de l'école néoclassique, qui, à sa différence, a accordé une place de choix à Dupuit dans son Panthéon théorique. 
maillage du territoire arrivait à son terme : celui de la rentabilité de la construction des dernières voies prévues par les plans successifs d'aménagement ferroviaire. La théorie de Dupuit est ainsi au cœur du débat sur l'« utilité » des voies ferrées d'intérêt local qui, au début des années 1890, oppose Armand Considère (1841-1914) à Clément Colson (18531939) qui vient d'être nommé à la chaire d'économie politique de l'École des Ponts.

Armand Considère avait publié en 1892 dans les Annales des Ponts un important mémoire sur «L'utilité des chemins de fer d'intérêt local », titre qui faisait manifestement référence à Dupuit, qu'il citait ${ }^{33}$. Colson critique ce mémoire dans la livraison suivante des Annales. Deux ans plus tard Considère répond à cette critique en prenant la théorie de Dupuit à témoin : "On nomme valeur d'un transport la valeur que lui assigne l'expéditeur, c'est-à-dire le prix dont il consent à le payer et qu'il ne veut pas dépasser. C'est une notion bien nette qui correspond à un fait réel ; c'est celle des économistes et de Dupuit, que nous avons adoptée dans notre premier mémoire. Il est certain que M. Colson l'admet également, bien qu'il ne l'ait pas dit avec une absolue netteté ${ }^{34}$. »

Or, il faut souligner que Considère mobilise ici Dupuit à rebours. Comme on l'a vu en effet, Dupuit avait développé sa mesure de l'utilité pour dénoncer l'exagération de la mesure de l'utilité publique des investissements en voie de transport qui résultait du modèle de Say et Navier. Considère au contraire s'appuie sur Dupuit pour montrer que l'évaluation des investissements ferroviaires par la profitabilité des entreprises est erronée, car elle sous-évaluerait leur utilité publique. Pour lui, le marché ne fournit pas une bonne évaluation de la valeur réelle, car il ne prend pas en considération ce qu'on appellera les " effets induits », soit les effets externes positifs qui résulteraient de l'ouverture d'une ligne. Une nouvelle fois, le calcul d'utilité vient ici non pas légitimer l'évaluation marchande, mais se substituer à elle. Il s'agit de dégager une autre légitimation à la construction de lignes que le montant, manifestement insuffisant, des recettes attendues. La question reste vive aujourd'hui, même si elle est souvent devenue défensive : il ne s'agit plus tant de légitimer l'extension du réseau, mais son maintien au moins partiel ${ }^{35}$.

33- A. Considère, "L'utilité des chemins de fer d'intérêt local ", Annales des Ponts et Chaussées, 1892, $1^{\text {er }}$ sem., p. 217-285 et 354-357.

34- A. Considère, «Utilité des chemins de fer d'intérêt local. Examen des observations formulées par M. Colson ", ibid., 1894, $1^{\text {er }}$ sem., p. 16-151 et 133-137, voir p. 97.

35- À l'exception bien sûr des cas du chemin de fer à grande vitesse et du réseau urbain. 


\section{Conclusion}

La principale leçon que l'on peut retenir de cette aventure théorique est l'apparent paradoxe qui veut que l'ingéniosité théorique de Dupuit en économie soit venue, précisément, de son engagement professionnel dans une économie routière, où l'évaluation marchande faisait défaut. Or, le très libéral Dupuit ne récusait aucunement, d'un point de vue philosophique, l'évaluation marchande. Au contraire, par son « expérience de pensée » du " prix maximum accepté », il la pousse en quelque sorte à son paroxysme. Dans cette nouvelle épistémologie économique, le marché est présent en permanence dans la tête des acteurs qui mesurent implicitement l'utilité au-delà du marché effectif. Telle est la grande modernité de la pensée économique de Dupuit.

Il faut souligner aussi que, chez Dupuit, la valeur reste liée à la demande solvable. L'interprétation de Considère est en ce sens un détournement, heureux, de sa théorie qui ne visait pas à mettre en évidence des effets au-delà du marché. Mais, par son principe de tarification, Dupuit cherchait, non sans une certaine naïveté théorique, à rendre solvable toute la demande. L'ultra-libéralisme le conduisait ainsi à un principe de justice sociale original ${ }^{36}$. Son héritage est donc complexe, tant du point de vue doctrinal qu'analytique. Ainsi, quand la théorie de Dupuit réapparaît dans les années 1890 chez les ingénieurs ferroviaires, c'est pour tenter de dépasser l'évaluation marchande ordinaire au nom du principe d'un intérêt général que le marché ne saurait instaurer. C'est chez des critiques de l'«horreur économique » que l'on retrouverait aujourd'hui semblable argument, pour sauver des lignes dans l'espace rural par exemple.

L'économie est-elle vraiment, comme on le pense en général, une « science du marché »? Ou n'a-t-elle finalement de sens que dans les pores, les limites, les apories du marché ? A cet égard, l’héritage du très libéral Dupuit apparaît bien plus complexe et contradictoire qu'il n'y paraît a priori.

36- Comme on l'a vu, l'éthique utilitariste de Dupuit le conduit à propos de la tarification à un argumentaire qui rejoint la doctrine de l'impôt progressif. Or la progressivité de l'impôt, qui fait partie des réformes exigées par Karl Marx et Friedrich Engels dans le Manifeste communiste de 1848, est en revanche majoritairement rejetée par la plupart des économistes libéraux du XIx ${ }^{e}$ siècle qui, de façon générale, se méfiaient de l'impôt sur le revenu. Ce n'est qu'après la guerre de 1914-1918 que ce principe s'imposera en France, de façon lente et limitée. 